\title{
Heat transfer analysis in stretching/shrinking rectangular fin with convection and radiation
}

\author{
Sharif Ullah ${ }^{1}$, Amir Ali $^{1}$, and Zia Din ${ }^{1}$ \\ ${ }^{1}$ University of Malakand
}

March 26, 2021

\begin{abstract}
The aim of this work is to enhance the heat transfer and study the efficiency of stretching/shrinking, radiating and rectangular fins. The effect of the dimensionless parameters, that is, radiation-conduction, convection-conduction stretching, thermogeometric parameters as well as the Peclet number, and surface temperature are investigated on the efficiency of stretching/shrinking and rectangular fins. The considered model is studied analytically using Differential Transform Method (DTM). The result is analyzed with the numerical solution for the accuracy of the semi-analytical solution, where good agreement is obtained. The impact of the considered parameters is studied numerically on the temperature distribution, fin's tip temperature, and the efficiency of the fin, where the combined effects of radiation and stretching/ shrinking enhance the system in the heat transfer with better efficiency. The shrinking of the fin with radiation increases the efficiency as compared to stretching with radiation is observed, which plays a significant role in mechanical engineering.
\end{abstract}

\section{Hosted file}

Streching_Rectangular_fin.pdf available at https://authorea.com/users/404237/articles/515497heat-transfer-analysis-in-stretching-shrinking-rectangular-fin-with-convection-andradiation 